Review article

\title{
Biochemical and imaging parameters in acid sphingomyelinase deficiency: Potential utility as biomarkers
}

\author{
Eline C.B. Eskes ${ }^{\mathrm{a}}$, Barbara Sjouke ${ }^{\mathrm{a}}$, Frédéric M. Vaz ${ }^{\mathrm{b}}$, Susan M.I. Goorden ${ }^{\mathrm{b}}$, \\ André B.P. van Kuilenburg ${ }^{\mathrm{b}}$, Johannes M.F.G. Aerts ${ }^{\mathrm{c}}$, Carla E.M. Hollak ${ }^{\mathrm{a}, *}$ \\ ${ }^{a}$ Amsterdam UMC, University of Amsterdam, Department of Endocrinology and Metabolism, Meibergdreef 9, 1105 AZ Amsterdam, The Netherlands \\ ${ }^{\mathrm{b}}$ Amsterdam UMC, University of Amsterdam, Department of Clinical Chemistry, Laboratory Genetic Metabolic Diseases, Gastroenterology \& Metabolism, Meibergdreef 9 , \\ 1105 AZ Amsterdam, The Netherlands \\ ${ }^{\mathrm{c}}$ Leiden Institute of Chemistry, University of Leiden, Department of Medical Biochemistry, Einsteinweg 55, 2333 CC Leiden, The Netherlands
}

\section{A R T I C L E I N F O}

\section{Keywords:}

Acid sphingomyelinase deficiency (ASMD)

Niemann-Pick disease type B (NPB)

Biomarkers

Clinical endpoints

Biochemical markers

Imaging parameters

\begin{abstract}
A B S T R A C T
Acid Sphingomyelinase Deficiency (ASMD), or Niemann-Pick type A/B disease, is a rare lipid storage disorder leading to accumulation of sphingomyelin and its precursors primarily in macrophages. The disease has a broad phenotypic spectrum ranging from a fatal infantile form with severe neurological involvement (the infantile neurovisceral type) to a primarily visceral form with different degrees of pulmonary, liver, spleen and skeletal involvement (the chronic visceral type). With the upcoming possibility of treatment with enzyme replacement therapy, the need for biomarkers that predict or reflect disease progression has increased. Biomarkers should be validated for their use as surrogate markers of clinically relevant endpoints. In this review, clinically important endpoints as well as biochemical and imaging markers of ASMD are discussed and potential new biomarkers are identified. We suggest as the most promising biomarkers that may function as surrogate endpoints in the future: diffusion capacity measured by spirometry, spleen volume, platelet count, low-density lipoprotein cholesterol, liver fibrosis measured with a fibroscan, lysosphingomyelin and walked distance in six minutes. Currently, no biomarkers have been validated. Several plasma markers of lipid-laden cells, fibrosis or inflammation are of high potential as biomarkers and deserve further study. Based upon current guidelines for biomarkers, recommendations for the validation process are provided.
\end{abstract}

\section{Introduction}

\section{1. $A S M D$}

Acid Sphingomyelinase Deficiency (ASMD), also known as Niemann-Pick disease types A and B, is a rare lysosomal storage disorder (LSD) caused by mutations in the sphingomyelin phosphodiesterase 1 (SMPD1) gene, leading to strongly decreased activity of acid sphingomyelinase (ASM, EC 3.1.4.12) [1-4]. The enzyme ASM is mainly present in lysosomes and catalyzes hydrolysis of sphingomyelin (SM) to ceramide and phosphocholine [5,6] (see Fig. 1). In ASMD, SM and its precursor lipids accumulate in lysosomes, especially in macrophages. These lipid laden macrophages, Niemann-Pick cells, can be found in several tissues, mainly the spleen, liver and lungs [1],

\footnotetext{
Abbreviations: 6MWT, 6 minute walk test; ALT, alanine aminotransferase; ASM, acid sphingomyelinase; ASMD, acid sphingomyelinase deficiency; AST, aspartate

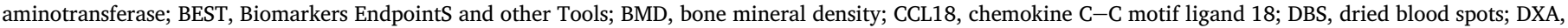

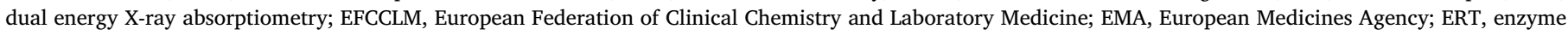

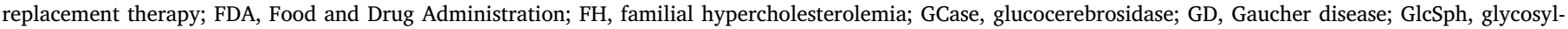

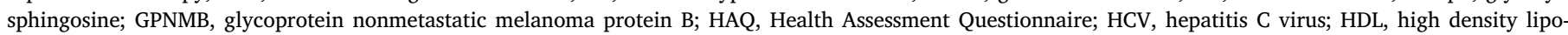

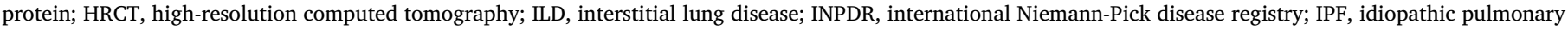

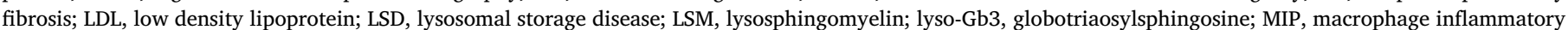

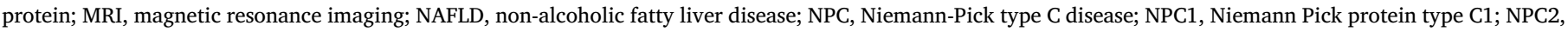

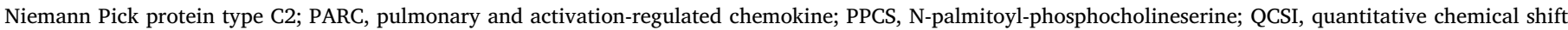
imaging; SF36, Short Form 36 Health Survey; SM, sphingomyelin; SMPD1, sphingomyelin phosphodiesterase 1

* Corresponding author at: Amsterdam UMC, University of Amsterdam, Department of Endocrinology and Metabolism, Meibergdreef 9, 1105 AZ Amsterdam, The Netherlands.

E-mail address: c.e.hollak@amc.nl (C.E.M. Hollak).
} 


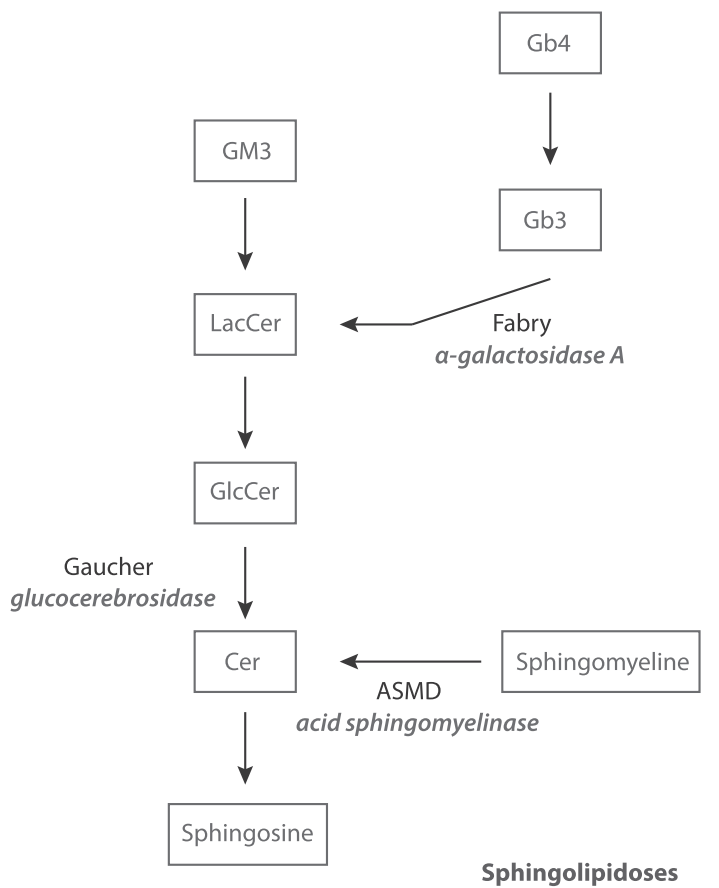

Fig. 1. Metabolic pathways of the degradation of sphingolipids.

Acid sphingomyelinase deficiency (ASMD) is caused by a deficiency of the enzyme acid sphingomyelinase. This hampers the conversion of sphingomyelin into ceramide. The metabolites are presented in the frames, enzymes are printed in italic, the sphingolipidoses are printed in black. Gb4: globoside, Gb3: globotriaosylceramide, GM3: monosialodihexosylganglioside, LacCer: lactosylceramide, GlcCer: glucocerebroside, Cer: ceramide.

leading to variable degrees of hepatosplenomegaly, cytopenia and pulmonary disease [1]. The prevalence of ASMD is estimated to be approximately 1 per 200.000 births [7,8]. Traditionally different subtypes are identified: ASMD type A, now more accurately referred to as the infantile neurovisceral form, is characterized by a relatively low residual ASM activity and shows a severe disease course with neurological involvement in young children, which is fatal before the age of three years $[9,10]$. On the other end of the spectrum is type $B$, better described as the chronic visceral form, which is characterized by absence of neurological involvement and instead, the presence of variable visceral symptoms. The intermediate form, traditionally called type A/ $B$, but now the chronic neurovisceral form, encompasses patients surviving early childhood, who have mild to severe neurological symptoms in addition to visceral symptoms [11].

The most common symptoms of the visceral phenotype are hepatosplenomegaly, thrombocytopenia and interstitial lung disease with decreased diffusion capacity. When disease progresses, liver failure and respiratory insufficiency can occur. Natural history studies indicate that the disease can be stable or show minimal progression over the course of years in adult patients [12-14].

Due to heterogeneity of symptoms, the clinical diagnosis can be difficult. The gold standard for diagnosis is demonstration of deficient ASM activity in leukocytes or fibroblasts. In case of a decreased enzyme activity, additional gene sequencing should be performed [15]. Over 180 mutations in the SMPD1 gene have been identified, but genotypephenotype correlations are not straightforward in most cases [16].

Already in the original case descriptions of patients suffering from the chronic visceral form of ASMD, the similarity to type 1 Gaucher disease (GD) was highlighted [17]. Gaucher disease is caused by a mutation in the GBA1 gene causing deficiency of the lysosomal enzyme glucocerebrosidase (GCase) (EC 3.2.1.45) [18,19]. Both diseases are characterized by accumulation of lipid-laden macrophages with quite similar overall appearance. However, the storage material in Gaucher cells and Niemann-Pick cells is distinct. As indicated previously, Niemann-Pick cells prominently accumulate SM, whereas Gaucher cells accumulate glucosylceramide, the simplest glycosphingolipid [20,21] (see Fig. 1). Although the clinical symptoms of type 1 GD and ASMD patients are quite similar, in GD bone disease and pain are more prominent than in ASMD. Lung disease and cirrhosis are more common in ASMD than GD.

\subsection{Enzyme replacement therapy}

At present, the mainstay of therapy is supportive care. Currently, a phase II/III clinical trial of enzyme replacement therapy (ERT, olipudase alfa, Sanofi Genzyme, MA, USA) in the chronic visceral form of ASMD is being conducted [22]. The enzyme ASM differs from other lysosomal enzymes like GCase by the fact that it is partially secreted and moreover able to degrade SM at the surface of cells [5]. Probably as a consequence of this, initial high doses of enzyme may trigger direct release of ceramide in the bloodstream, which induces a pro-inflammatory response [23]. Hence, a careful dosing study was performed in a phase I trial, in eleven patients who each received a single injection of olipudase alfa at different doses $(0.03-1.0 \mathrm{mg} / \mathrm{kg})[24,25]$. The phase Ib study consisted of a within-patient dose escalation program over 26 weeks and was conducted in five patients who all reached the maximum dose of $3.0 \mathrm{mg} / \mathrm{kg}[26,27]$. Results of the extended phase $1 \mathrm{~b}$ trial were published after 30 months of treatment. Four patients who continued at a dose of $3.0 \mathrm{mg} / \mathrm{kg}$, and one patient at an adjusted dose of $1.0 \mathrm{mg} / \mathrm{kg}$ [28]. All five patients showed promising results, i.e. decrease of hepatosplenomegaly, improvement of pulmonary diffusion capacity and ameliorated lipid profiles. Indeed, since lipid-laden macrophages are the primary storage cells in ASMD, clinical benefit by correction of these cells by ERT seems feasible as indicated by the success of this approach with GD $[29,30]$.

It should be noted that two out of the five patients enrolled in the phase Ib trial had normal diffusion capacity at baseline [28]. All patients included in both the phase I and Ib trial were diagnosed with the chronic visceral form of ASMD and had no neurological symptoms $[24,26]$.

\subsection{Biomarkers}

Biomarkers are defined as 'characteristics that are objectively measured and evaluated as an indicator of normal biological processes, pathogenic processes, or pharmacologic responses to a therapeutic intervention' [31]. Biomarkers, when properly validated, can function as surrogate endpoints and thus shorten clinical trials [32,33]. Moreover, they can replace invasive, time consuming or costly procedures in daily practice. Biomarkers are often biological molecules measured in easily accessible specimens such as plasma and urine or tissue, but they can also be parameters measured in medical procedures for instance imaging techniques.

The European Medicines Agency (EMA) and the Food and Drug Administration (FDA), trying to create order in the expanding field of biomarker research, have issued guidelines for the registration of biomarkers mainly to ensure proper use as surrogate endpoints in clinical trials $[34,35]$. However, clear recommendations are lacking.

There are many pitfalls in the current biomarker pipeline [36]. A major issue is the lack of proper evaluation both before and after clinical implementation. A more effective approach would be to focus on the clinical use of a specific biomarker and thus starting with defining the patient population and the clinical outcome(s) the biomarker has to reflect or predict [36]. In line with this, a working group of the European Federation of Clinical Chemistry and Laboratory Medicine (EFCCLM) has released a 14-item checklist, which guides researchers through the process of identifying and implementing a new biomarker [37].

In ASMD little is known about biochemical or imaging markers that 
can be used as surrogates for hard clinical endpoints such as survival, organ failure or impaired quality of life. Especially for slowly progressive, chronic diseases such as ASMD, the availability of substitutes is necessary to be able to assess the impact of specific therapies since clinical trials are usually limited to 6 or 12 months of initial follow-up. In the search of biomarkers for ASMD, GD can serve as an example because the macrophage is the central storage cell in both disorders. Gaucher cells have been studied in more detail in the past and are mainly viable alternatively activated macrophages that secrete specific proteins into the circulation, resulting in elevated plasma levels [38]. For example, the elevated plasma proteins in GD patients (chitotriosidase and chemokine $\mathrm{C}-\mathrm{C}$ motif ligand 18, better known as CCL18) are markers of body burden on Gaucher cells (reviewed in [39]) and also reported as plasma markers of Niemann-Pick cells (see section 3.6.2).

The purpose of this review is to summarize known biochemical and imaging markers of visceral manifestations of ASMD in the context of hard clinical endpoints and discuss their validity as surrogate endpoints. Markers of neurological disease are of high importance, but are not included here since ERT is unable to ameliorate central nervous system symptoms [26]. Furthermore, we propose several markers that could be of additional value. Considering the heterogeneity of the course of disease in ASMD, the chance of finding one biomarker that reflects disease activity and predicts the course of disease is extremely small. Therefore, possible biomarkers for the different organ manifestations are discussed.

\section{Methods}

\subsection{Search strategy}

A literature search (see Fig. 2) was performed using the PubMed

(((((Niemann Pick) OR ((("Niemann-Pick Disease, Type B"[Mesh]) OR "Niemann-Pick Disease, Type A"[Mesh]) OR "Niemann-Pick Diseases"[Mesh])) OR acid sphingomyelinase deficien*) OR ASMD)) AND (((((biomarke*) OR "Biomarkers"[Mesh]) OR imagin*) OR histolog*) OR biochemi*)

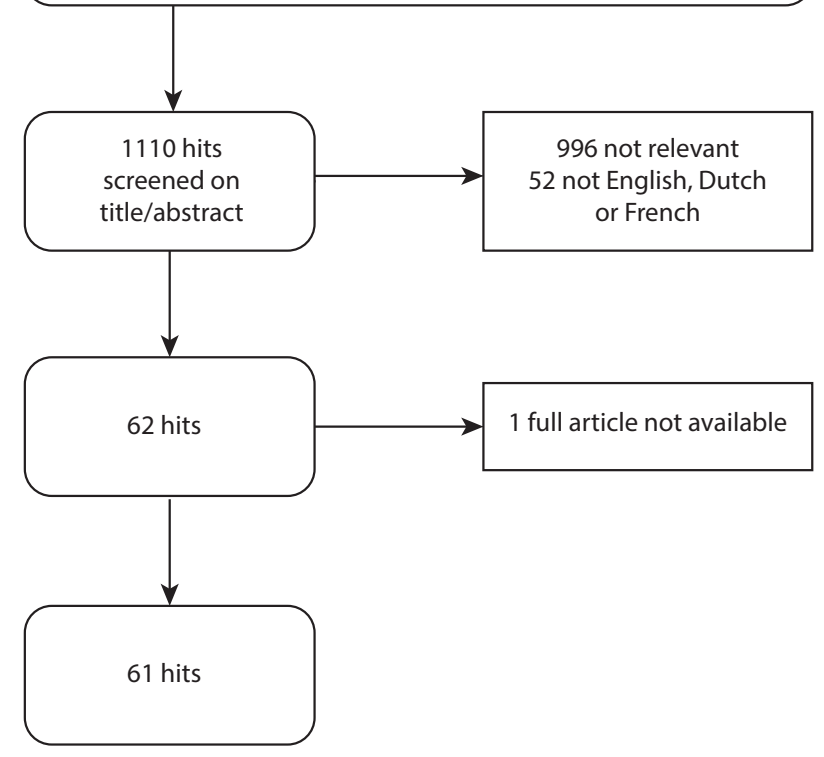

Fig. 2. Search strategy in PubMed.

The above mentioned search terms were used in the PubMed database and resulted in 1110 hits. Titles and abstracts were screened for relevance regarding biomarkers in ASMD, 996 did not meet this criterion. 52 publications were non English, Dutch or French. 1 of the remaining 62 publications was not accessible. This resulted in 61 publications included in this review. database by combining the key words: 'Niemann Pick disease', 'acid sphingomyelinase deficiency' and their variations with 'biomarkers' and variations on the terms 'imaging', 'histology' and 'biochemical'. This yielded 1110 hits. Title and abstract were screened for research regarding biomarkers or diagnostic tools for ASMD combined with clinical endpoints as defined in section 2.2, which led to exclusion of 996 publications and an additional 52 that were not in English, Dutch or French. Full text of 61 of the remaining 62 publications was available for review.

\subsection{Identification of clinical endpoints}

The chronic visceral form of ASMD is a slowly progressive disease with long stable periods. Since clinical events are scarce, surrogate endpoints that can substitute for hard endpoints are of value. In this section, non-neurological clinical endpoints in ASMD are discussed and defined sorted by organ system (see Fig. 3 ).

Liver disease in ASMD progresses via elevation of liver enzymes to fibrosis and eventually cirrhosis. Cirrhosis is associated with deterioration of liver function, portal hypertension and increased risk of hepatocellular carcinoma [40]. Morbidity and mortality of non-ASMD related liver cirrhosis are substantial [41]. Five-year survival of patients with liver cirrhosis due to non-alcoholic fatty liver disease (NAFLD) with cirrhosis is similar to that observed in patients with liver cirrhosis due to hepatitis C virus (HCV) and is approximately 75\% [42].

Lung disease in ASMD consists of restrictive lung disease, decreased diffusion capacity and interstitial lung disease (ILD) with signs of pulmonary fibrosis, all as a result of SM accumulation [43,44]. Since ILD composes a large group of different diseases, linking the scientific knowledge of the pathogenesis of patients with ILD to ASMD is difficult. ILD is associated with an increased mortality rate [45]. Important prognostic markers of idiopathic pulmonary fibrosis (IPF) are a decrease in diffusion capacity and a decrease of exercise capacity [46].

The third important group of clinical events in ASMD is related to splenomegaly and contains hemorrhage and spleen infarction, which can both require splenectomy. Hemorrhage is mainly caused by thrombocytopenia due to hypersplenism. Clinical events related to hemorrhage that have been described in ASMD are recurrent epistaxis, subdural hematoma, hematemesis, hemoptysis and haematothorax. Several cases where splenic bleeding led to splenectomy have been described [47-51]. Splenomegaly can also cause infarction in the spleen, possibly leading to necrosis and thus requiring splenectomy as described in one case report [52]. Long-term outcomes in ASMD patients after splenectomy have not been systematically described, but SM storage shifts may increase the storage burden in other organs [49,51]. This is exemplified in Gaucher disease, where splenectomy leads to aggravation of liver disease, bone disease and an increased risk of developing malignancies [53,54].

Since patients often show an atherogenic lipid profile with low high density lipoprotein (HDL) and high low density lipoprotein (LDL), patients are at higher risk of cardiovascular events [55-57]. One publication that focuses on morbidity and mortality in ASMD patients reports a few patients suffering from cardiac complaints [56]. Moreover, two sisters with cardiac complications and ASMD were described [57] and a patient who presented with cor pulmonale considered secondary to pulmonary involvement of ASMD [58]. Thus, we consider cardiovascular events to be an important clinical endpoint as well.

Skeletal involvement is present in ASMD, but its extensiveness is reported variably. Events that have been described in natural history studies are pathological fractures, decreased bone mineral content and decreased bone mineral density $[11,12,55,59]$.

Lastly, biochemical markers are of particular interest in the search for biomarkers, since they might reflect general burden of disease. Several plasma markers reflect disease activity that is an effect of general SM accumulation and not organ specific.

The aforementioned events are all objective and can be measured 


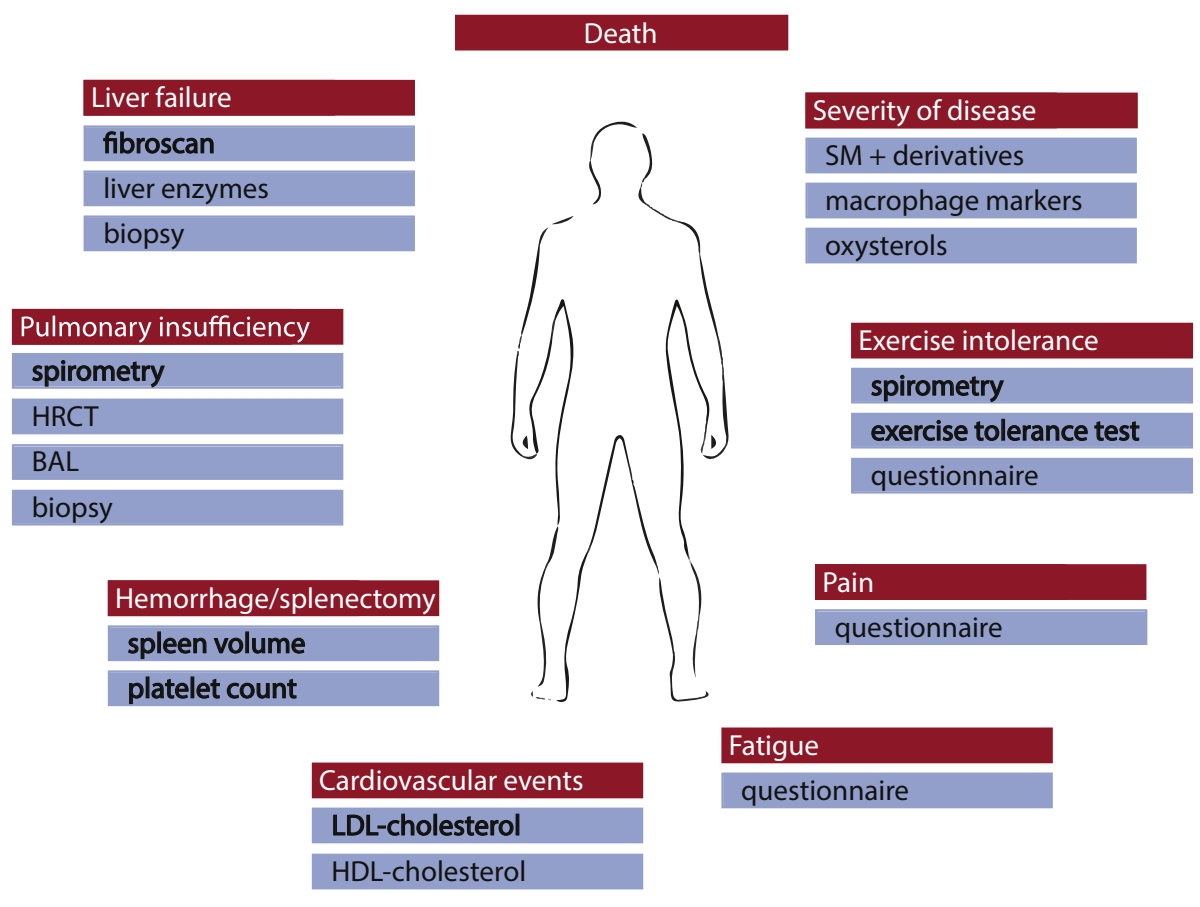

Fig. 3. Clinical endpoints in ASMD and where applicable their associated biomarkers.

See text for each section; i.e. liver transaminases can be normal and do not exclude liver fibrosis/cirrhosis (section 3.1). The most promising markers according to Table 1 are depicted in bold. Questionnaires do not meet the definition of a biomarker, but are depicted here because they are the best way to structurally assess quality of life. BAL: broncho-alveolar lavage, HRCT: high-resolution computed tomography, SM: sphingomyelin, 6MWT: 6 minute walk test, DXA: dual energy X-ray absorptiometry, QCSI: quantitative chemical shift imaging. and assessed. Quality of life on the other hand is a subjective parameter and does not strictly fulfill the definition of a biomarker. Therefore, this endpoint is not included in the results section.

\subsection{Analysis}

Biomarkers in ASMD that are valuable for monitoring patients should (a) reflect disease activity and (b) predict the likelihood of clinical events and hence in time aid in decision making about initiation of treatment. With the glossary of the Biomarkers, EndpointS and other Tools (BEST) Resource [32] and the checklist of the EFCCLM [37] as starting points as well as the characteristics of an ideal biomarker of LSDs as proposed by Cox [33] three important hallmarks of biomarkers in ASMD were defined: (a) the link to the pathophysiological process should be clear, (b) levels should change in response to effective therapy and (c) correlation with clinical events as defined in section 2.2 should be established. Each marker received a tentative validation score based on these criteria, with a maximum of +++ . Procedures considered too invasive scored -. Markers that are very promising based on potential correlation to a clinical endpoint scored an additional + .

When data on the discussed biomarkers in ASMD are limited, related diseases are described. These include Gaucher disease, NiemannPick type C (NPC), where a primary defect in cholesterol transport results in secondary SM accumulation, and Fabry disease, a sphingolipidosis with accumulation of globotriaosylceramide (see Fig. 1).

\section{Results}

The potential biomarkers as found in the reviewed publications are depicted in Table 1, together with an assessment of their validity. Fig. 4 presents an overview of the biochemical markers and their interdependence.

\subsection{Markers regarding liver disease}

SM storage in the liver is a direct consequence of the deficiency of ASM and is mainly observed in the liver-specific macrophages, the Kupffer cells $[25,27,60]$. The storage pattern of SM seems to reflect disease severity: biopsies of patients with lower residual enzyme activity showed higher amounts of SM and accumulation in hepatocytes besides Kupffer cells [25,60]. The phase Ib trial of ERT with olipudase alfa showed significant reduction of SM storage after 26 weeks of treatment [27]. However, assessing the presence of fibrosis or cirrhosis in these liver biopsies may be even more important, since this directly reflects one of the clinical endpoints as defined in section 2.2. Fibrosis is also associated with an SM storage pattern in both Kupffer cells and hepatocytes [25]. Interestingly, after 26 weeks of ERT 3 patients showed an increase in fibrosis score and 2 showed no change [27].

Other techniques to detect early stages of fibrosis in tissue are of interest because ideally, they would replace invasive and risky biopsies. Transient elastography (also known as fibroscan) is broadly used in monitoring the progression of fibrosis in patients with HCV and proved to be a solid alternative for liver biopsies [61]. Fibroscans have been studied in Gaucher disease. Webb et al. described fibroscans and shear wave elastography as useful techniques to measure spleen and liver stiffness and thereby distinguish Gaucher patients from healthy subjects and patients with cirrhosis [62]. Fibroscans are not described in relation to ASMD, but are easy to perform and promising for detecting fibrosis in ASMD patients. Therefore they are recommended by a panel of international ASMD experts [11]. The expected correlation to liver fibrosis resulted in an additional + on tentative validation score (see Table 1).

Liver enzymes in plasma are often mildly elevated in ASMD, which can be explained by enlargement of the liver and a mild inflammation reaction due to accumulation of SM in the liver. However, it is not elucidated whether increase of plasma aminotransferase (ALT/AST) levels reflects development of liver fibrosis or cirrhosis in ASMD. In the phase Ib trial of olipudase alfa, all five patients had ALT and AST levels within the normal ranges, except for a transitory rise in one patient, and therefore the effect of ERT could not be assessed [28]. In NAFLD it has been demonstrated that $30-60 \%$ of the patients with a biopsy-confirmed NAFLD have normal AST levels [63]. NAFLD patients with normal ALT levels have the same risk of disease progression as NAFLD patients with elevated ALT levels [64]. In line with these findings, an ASMD patient with normal plasma liver enzyme levels was diagnosed with liver cirrhosis [65]. Thus, elevation of plasma aminotransferases could indicate liver fibrosis, but normal levels do not exclude this. 
Table 1

Potential biomarkers for ASMD.

\begin{tabular}{|c|c|c|c|c|c|}
\hline Potential biomarker & Intervention & $\begin{array}{l}\text { Link to the pathological process } \\
\text { established? }\end{array}$ & $\begin{array}{l}\text { Response to ERT } \\
\text { demonstrated? }\end{array}$ & $\begin{array}{l}\text { Relation to clinical endpoint(s) } \\
\text { established? }\end{array}$ & $\begin{array}{l}\text { Tentative validation } \\
\text { score }\end{array}$ \\
\hline \multicolumn{6}{|l|}{ Liver involvement } \\
\hline SM accumulation & Liver biopsy & yes & yes & no & -4 \\
\hline Fibrosis & Liver biopsy & yes & no $^{1}$ & yes & -4 \\
\hline Fibrosis & Fibroscan & yes & $\mathrm{no}^{2}$ & yes & ++ \pm \\
\hline Fibrosis & SWE & yes & $\mathrm{no}^{2}$ & yes & ++ \\
\hline Liver transaminases & Venipuncture & yes & no $^{3}$ & no & + \\
\hline \multicolumn{6}{|l|}{ Lung involvement } \\
\hline SM accumulation & Lung biopsy & yes & $\mathrm{no}^{2}$ & no & -4 \\
\hline Fibrosis & Lung biopsy & yes & $\mathrm{no}^{2}$ & yes & -4 \\
\hline SM accumulation & BAL & yes & $\mathrm{no}^{2}$ & no & -4 \\
\hline Parameters of ILD & HRCT & yes & yes & no & ++ \\
\hline Diffusion capacity & Spirometry & yes & yes & yes & +++ \\
\hline \multicolumn{6}{|l|}{ Spleen involvement } \\
\hline Spleen volume & MRI/CT & yes & yes & yes & +++ \\
\hline Platelet count & Venipuncture & yes & yes & yes & +++ \\
\hline \multicolumn{6}{|l|}{ Cardiovascular risk } \\
\hline HDL-cholesterol & Venipuncture & yes & yes & no & ++ \\
\hline LDL-cholesterol & Venipuncture & yes & yes & yes & +++ \\
\hline \multicolumn{6}{|l|}{ Skeletal involvement } \\
\hline Bone marrow fat fractions & QCSI & yes & $\mathrm{no}^{2}$ & no & + \\
\hline Bone mineral density & DXA & yes & no ${ }^{3}$ & yes & ++ \\
\hline \multicolumn{6}{|l|}{ Burden of disease } \\
\hline SM & Venipuncture & yes & $\mathrm{no}^{3}$ & no & + \\
\hline LSM & Venipuncture & yes & yes & no & ++ \pm \\
\hline LSM-509/PPCS & Venipuncture & yes & $\mathrm{no}^{2}$ & no & + \pm \\
\hline Ceramide & Venipuncture & yes & $\mathrm{no}^{3}$ & no & + \\
\hline Chitotriosidase & Venipuncture & yes & yes & no & ++ \\
\hline CCL18 & Venipuncture & yes & yes & no & ++ \\
\hline GPNMB & Venipuncture & yes & $\mathrm{no}^{2}$ & no & + \\
\hline MIP-1 $\alpha$ & Venipuncture & no & $\mathrm{no}^{2}$ & no & - \\
\hline MIP-1 $\beta$ & Venipuncture & no & $\mathrm{no}^{2}$ & no & - \\
\hline Cholestane- $3 \beta, 5 \alpha, 6 \beta$-triol & Venipuncture & yes & $\mathrm{no}^{2}$ & no & + \\
\hline 7-ketocholesterol & Venipuncture & yes & $\mathrm{no}^{2}$ & no & + \\
\hline Walked distance & 6MWT & yes & $\mathrm{no}^{2}$ & yes & ++ \pm \\
\hline
\end{tabular}

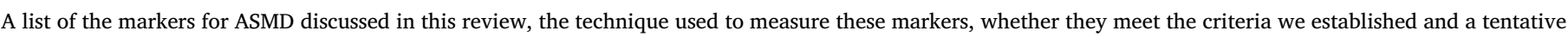

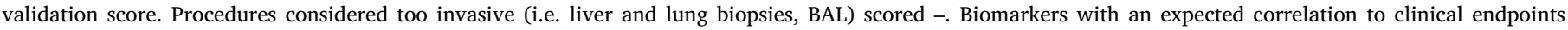
received an extra + (i.e. fibrosis measured with fibroscan, LSM, LSM-509 and 6MWT, additional + underlined in table).

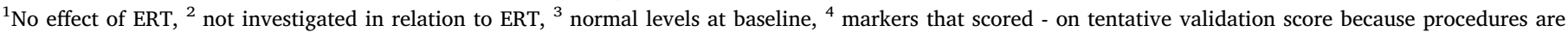
considered too invasive.

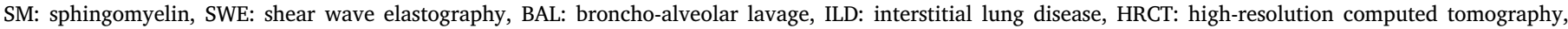

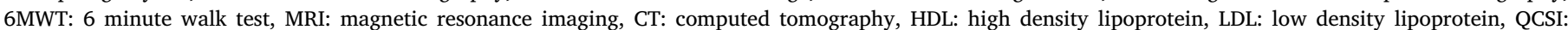

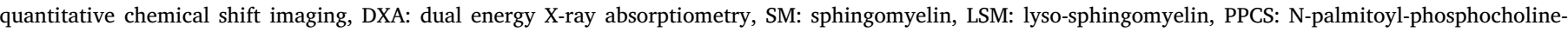
serine, CCL18: chemokine (C-C motif) ligand 18, MIP: macrophage inflammatory protein.

\subsection{Markers regarding lung disease}

There are several markers applicable for monitoring lung involvement in ASMD patients. Histological tests are performed to assess SM accumulation as primary effect of decreased ASM activity where inflammation and fibrosis are secondary effects. Lung biopsies show lipid laden cells, predominantly foamy macrophages located in the alveolar spaces and walls and to a lesser extent in the interstitium [43,66-68]. Inflammation of the interstitium and fibrosis are observed in varying degrees $[43,66,67]$. Broncho-alveolar lavage fluid shows foamy macrophages $[43,66,67,69]$. Effect of ERT on these markers has not been investigated.

High-resolution computed tomography (HRCT) is currently the most reliable technique to investigate (early) signs of ILD as a result of ASMD. Common findings in ASMD are a ground glass pattern, thickened interlobular septa and intralobular lines [43,44,67,70-73]. Several studies showed that both HRCT and X-ray of the lungs display abnormalities indicating interstitial lung disease, but findings often do not correlate with impairment of lung function or clinical symptoms $[66,70,74,75]$. The area of affected lung tissue measured on HRCT decreased after 30 months of ERT [28].
Spirometry is used to assess lung volumes and diffusion capacity. Spirometry in ASMD patients generally shows normal or decreased lung volumes with a decreased diffusion capacity, indicative of restrictive pulmonary disease [74]. There is no clear correlation between pulmonary involvement on HRCT and pulmonary function measured by spirometry. This was illustrated by Mendelson et al.: in a cohort of 52 patients some patients showed severe ILD on HRCT, but normal diffusion capacity, while other patients had moderate to severe decrease in diffusion capacity, but showed none to mild ILD [70]. The phase Ib trial showed encouraging results regarding diffusion capacity; the 3 patients with diffusion capacity impairment in the severe range showed the greatest improvement of approximately 40\% (2 patients) and 95\% (1 patient). Patient reported outcomes on fatigue and pain did not change [28].

\subsection{Markers regarding spleen involvement}

Splenomegaly is a distinct feature of ASMD, caused mainly by SM accumulation. Spleen volume measured by magnetic resonance imaging (MRI) in 59 patients was $11.1 \pm 5.7$ multiples of normal [75]. McGovern et al. considered spleen volume to be a possible biomarker in 
A

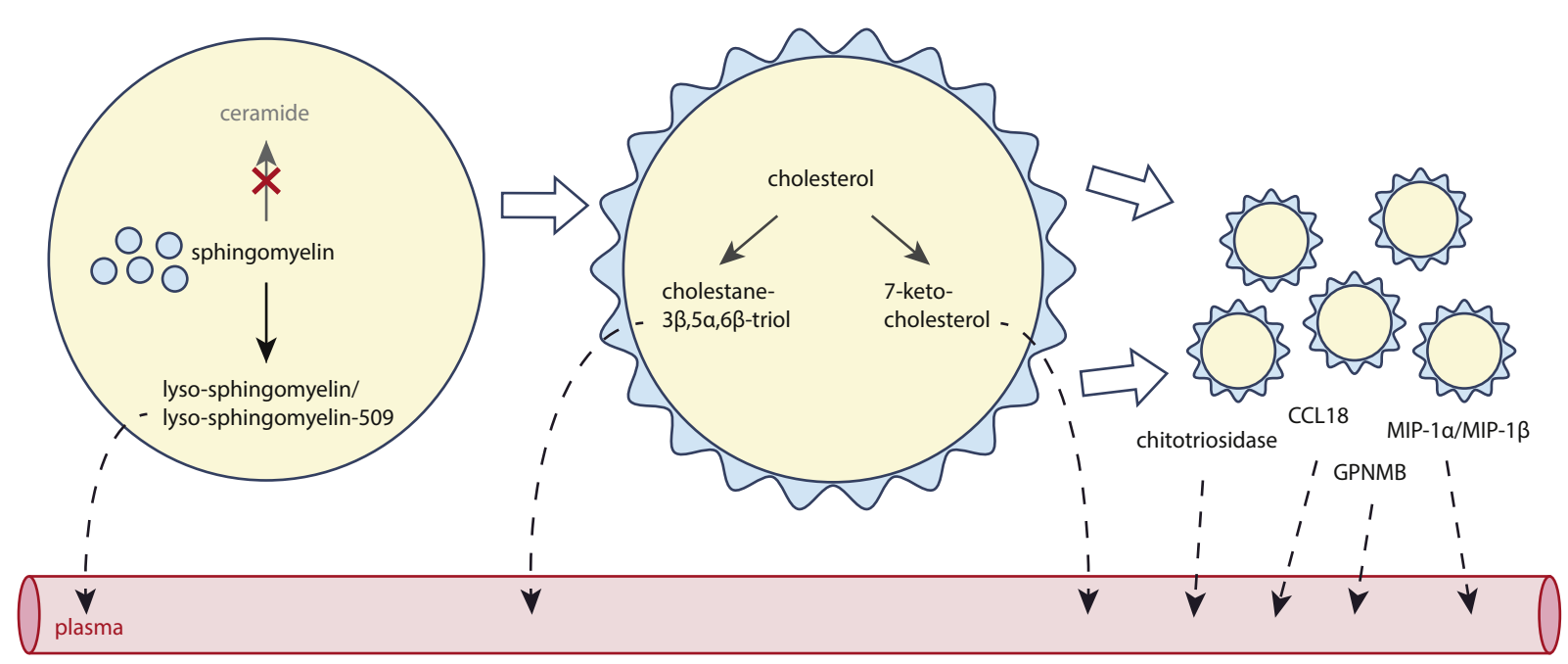

Fig. 4. Biochemical biomarkers in ASMD and their interdependence.

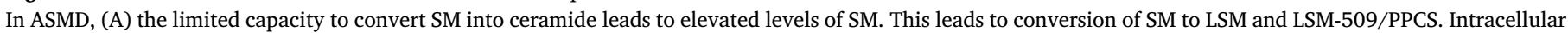

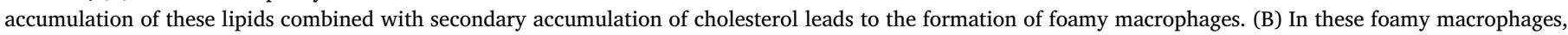

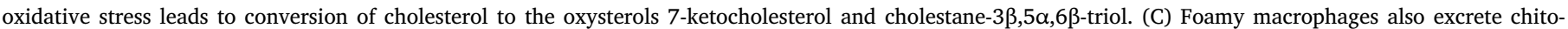

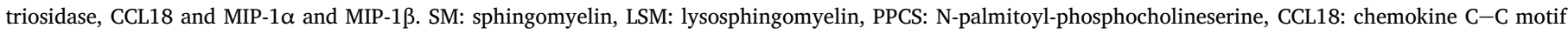
ligand 18, MIP: macrophage inflammatory protein.

ASMD. They demonstrated a positive correlation between spleen volume and liver volume and triglyceride levels, and a negative correlation with HDL levels, hemoglobin levels, white blood cell count and height [75]. Spleen volume decreased after ERT as observed in the phase Ib trial $[26,28]$.

In ASMD, low platelet count caused by splenomegaly is commonly found. Platelet count is mostly mildly decreased or low in the normal range $[12,13,56,75,76]$ and seems to worsen over time [12,13,76]. Platelet counts ameliorated over the course of the 30 month phase Ib study of olipudase alfa [28]. Correlation with clinical events in ASMD has not been established, although the correlation between thrombocytopenia and risk of bleeding is known in the general population [77].

\subsection{Markers regarding cardiovascular risk}

ASMD patients have a disruptive lipid profile with high plasma total cholesterol and LDL levels and low HDL levels [78]. The proposed explanation for this is that ASM deficiency leads to secondary inhibition of the Niemann Pick protein type C 1 (NPC1) and 2 (NPC2) which hampers cholesterol efflux via HDL and stimulates endogenic cholesterol production and LDL uptake. As mentioned earlier ASMD patients are presumably at a higher risk for cardiovascular disease [55-57]. In familial hypercholesterolemia (FH), patients have mutations in various genes leading to elevated plasma LDL levels. Since ASMD and FH are both inherited diseases, plasma LDL levels are elevated from birth and patients are similarly exposed to high levels, although levels of FH patients are more strikingly elevated. On the other hand, FH patients show HDL levels in the normal range [79].

Plasma HDL levels are decreased in ASMD patients, but the clinical consequences of these low HDL plasma levels are unclear. In the general population, HDL is regarded to be a predictor of cardiovascular risk, but causal relations between low HDL levels and cardiovascular events remain to be established [80]. In Gaucher disease, decreased HDL levels are not related to increased cardiovascular risk, although it should be noted that Gaucher patients have mildly decreased LDL levels as opposed to ASMD patients [81]. Lipid profiles of ASMD patients ameliorated after ERT as established in the phase Ib trial $[26,28]$.

\subsection{Markers regarding skeletal involvement}

In ASMD, decreased bone marrow fat fractions measured with quantitative chemical shift imaging (QCSI) have been described, presumably due to infiltration of foamy macrophages in bone marrow [12]. Fat fractions measured with QCSI are used in monitoring skeletal disease in Gaucher patients and increase significantly in response to ERT [82-84]. To assess bone mineral density (BMD), dual energy X-ray absorptiometry (DXA) scans can be performed. However, patients in the phase Ib ERT trial had normal BMD at baseline [28].

\subsection{Markers regarding burden of disease}

\subsubsection{Sphingomyelin and its derivatives}

In ASMD, the degradation of SM to ceramide is impaired, which leads to accumulation of SM. SM levels are not elevated in plasma or urine because of its hydrophobic nature [85]. Accumulated SM is converted into lysosphingomyelin (LSM), its deacylated form, by acid ceramidase. LSM is strongly elevated in plasma and dried blood spots of ASMD patients [85-91].

An analog of LSM, that until recently was called LSM-509, was also identified as a possible biomarker for ASMD since strongly elevated plasma levels were established in ASMD [86-90,92]. Recent findings show that LSM-509 belongs to a new class of lipids: N-acyl-O-phosphocholineserines with LSM-509 being N-palmitoyl-phosphocholineserine or PPCS [93]. In contrast to plasma samples, LSM-509/PPCS levels in dried blood spots (DBS) of ASMD patients show overlap with healthy subjects [86]. Although DBS are frequently used because they can easily be stored and shipped, analysis of LSM-509/PPCS in plasma is preferred over DBS. Two patients with a mild phenotype were reported to have normal or mildly elevated levels of LSM and LSM-509/ PPCS, which suggests a correlation with disease burden [86,88]. LSM decreased after 30 months of ERT [28].

In several LSDs the deacylated forms of sphingolipids are used to monitor disease burden in patients, since these metabolites are abundant in plasma. Examples are glycosylsphingosine (GlcSph) in Gaucher disease and globotriaosylsphingosine (lyso-Gb3) in Fabry disease, both formed by deacylation of the accumulating sphingolipids by acid 
ceramidase [94-96]. Of note, evidence has been presented that deacylated sphingolipids (sphingoid bases) may exert toxic effects. For example, excessive lyso-Gb3 in Fabry patients is thought to cause smooth muscle cell proliferation and uncoupling of the enzyme endothelial NOS, thus contributing to vasculopathy $[94,97]$. In addition, it has been linked to podocyturia, fibrosis and damage to nociceptive neurons [98-101]. In the case of Gaucher disease, excessive GlcSph has been proposed to cause $\alpha$-synuclein oligomerization, osteopenia, complement cascade activation and inflammation [102-106]. It will be of interest to investigate whether LSM in ASMD equally exerts toxic effects and whether its plasma levels can be related to risks for specific symptoms. As a biomarker, we expect that LSM and LSM-509/PPCS may prove to be sensitive and correlate to clinical endpoints (additional "+" on tentative validation score, see Table 1).

Ceramide can be produced by ASM via hydrolysis of SM but also via the novo synthesis and via hydrolysis of glucosyl- or galactosylceramides. It is involved in inflammatory pathways and causes apoptosis [107]. Plasma levels are normal or slightly elevated in ASMD patients, which is probably due to degradation of accumulated sphingolipids by other sphingomyelinases. Treatment of a patient with olipudase alfa resulted in transiently elevated levels of plasma ceramide [24,26]. At baseline of the phase I trial, ceramide levels of ASMD patients were normal, over the course of 26 weeks of ERT they decreased after every infusion and plateaued after 14 weeks $[24,26]$.

\subsubsection{Macrophage markers}

Several macrophage markers are elevated in plasma of ASMD patients: this rise reflects the intracellular lipid accumulation, which may lead to activation of macrophages and is also present in Gaucher disease, hence the overlap of the markers that are discussed in this section. Chitotriosidase is an enzyme that is produced by activated macrophages, therefore its plasma activity levels are elevated in several LSDs including ASMD [108]. Chitotriosidase has been extensively studied in Gaucher disease. The chitinase is secreted by Gaucher cells and its activity is $>600$ fold increased in plasma of Gaucher patients [109]. As such, it seems to directly reflect the disease burden [110]. Upon effective treatment, a rapid decrease in chitotriosidase plasma activity can be appreciated, as well as recurrence when treatment is interrupted, making it a useful marker to monitor therapy in Gaucher patients [109-111]. In ASMD, plasma levels of chitotriosidase are moderately increased [75,90,111-116] and do not overlap with levels as observed in Gaucher patients [90,112,114-116]. In the phase Ib trial of olipudase alfa, a decrease of chitotriosidase activity in plasma was observed [28].

A pitfall is that approximately $5 \%$ and $35 \%$ of the population is homozygous or heterozygous, respectively, for a 24 nucleotide duplication in the gene coding for chitotriosidase, resulting in absence of or decreased activity [117]. In this group of patients, CCL18 can serve as a biomarker [116]. The chemokine CCL18, also named pulmonary and activation-regulated chemokine (PARC), is excreted by lipid laden cells as well and seems to reflect alveolar macrophage activation [118]. Plasma levels are elevated in Gaucher patients, and decrease following treatment, in a manner comparable to the decrease of chitotriosidase after start of ERT [119,120]. Plasma levels of CCL18 are also elevated in ASMD patients, but response to therapeutic intervention has not been reported yet [113,120].

Glycoprotein nonmetastatic melanoma protein B (GPNMB) is a glycoprotein that is also secreted by activated macrophages [121]. Elevated plasma levels have been found in Gaucher and NPC mice [122-124]. In Gaucher mice, GPNMB is shown to correlate with chitotriosidase and CCL18 [122]. In Gaucher patients a correlation between plasma levels and disease severity was established [123]. It has not been studied in relation to ASMD, but seems a promising marker of macrophage activation as occurs in ASMD.

The chemokines macrophage inflammatory protein (MIP)- $1 \alpha$ and MIP-1 $\beta$, also referred to as CCL3 and CCL4, are elevated in ASMD and several other LSDs, although little is known about the mechanism leading to these increased levels. MIP-1 $\alpha$ is homologous to CCL18/ PARC: both cytokines from the C-C-chemokine family [125]. Plasma levels of MIP- $1 \alpha$ and MIP- $1 \beta$ decreased after start of ERT in Gaucher patients $[126,127]$. Despite their similarity, MIP-1 $\alpha$ and MIP-1 $\beta$ levels correlate poorly with chitotriosidase and CCL18 levels $[126,128]$. In ASMD knock out mice, MIP-1 $\alpha$ decreased after ERT [129]. While these MIPs have been proposed to relate to bone disease in Gaucher disease, no relation with clinical manifestations in ASMD has been reported. Whether other pro- and anti-inflammatory markers can serve as biomarkers in ASMD needs to be further explored.

\subsubsection{Oxysterols and bile acids}

ASMD and Niemann-Pick type C (NPC) share overlapping pathophysiological processes: the accumulation of sphingomyelin and its precursors caused by decreased activity of ASM leads to similar impediment of the NPC1 and NPC2 proteins, which are primarily defective in NPC. In NPC, it has been demonstrated that cholesterol accumulation induces oxidative stress, which results in the conversion of cholesterol into oxysterols [130-132]. Cholestane-3 $\beta, 5 \alpha, 6 \beta$-triol and 7-ketocholesterol, both oxysterols, are used as diagnostic markers in NPC; although levels are not elevated in all patients, the presence of elevated plasma levels is used to confirm the diagnosis [131,133-138]. In ASMD, plasma levels of these oxysterols are elevated as well, probably via a similar process caused by oxidative stress. Several studies showed significantly increased plasma levels in ASMD patients, without overlap with other LSDs $[88,90,130,139]$. It is possible to measure plasma levels of oxysterols in a short amount of time [130,134,137,140] and using this method for diagnosis is reported to be less costly, yet more effective in establishing a diagnosis than performing clinical examination of NPC patients [141]. In addition, these oxysterols did not show a correlation with severity scores of ASMD patients [92]. The effect of ERT on plasma oxysterol levels in ASMD patients has not been described. In patients with NPC treated with miglustat, cholestane$3 \beta, 5 \alpha, 6 \beta$-triol levels were significantly lower than those of untreated NPC patients [142]. A disadvantage of 7-ketocholesterol is that it is unstable after sample collection at room temperature but increases in concentration when stored, this is not the case for cholestane- $3 \beta, 5 \alpha, 6 \beta$ triol $[90,143]$.

It is hypothesized that in NPC the accumulation of oxysterols leads to altered bile acid formation. Elevated levels of the bile acids $3 \beta, 5 \alpha, 6 \beta$-trihydroxycholanic acid and $3 \beta$-sulfooxy-7 $\beta$ - $N$-acetylglucosaminyl-5-cholen-24-oic acid have been described in NPC patients. In ASMD, only elevation of $3 \beta, 5 \alpha, 6 \beta$-trihydroxycholanic acid has been described [144-150].

\subsubsection{Markers regarding exercise tolerance}

It is useful to gain insight into patients' tolerance to exercise, since this comprises pulmonary as well as cardiac function, stamina and functioning of the musculoskeletal system. The exercise protocol that is most described in patients with ASMD is the 6 minute walk test (6MWT). It has only been investigated in a few patients but they appeared to perform worse than healthy subjects and results seem to deteriorate over time [12]. This is in line with the expectation that the 6MWT, originally developed as an easy test to capture changes in cardiopulmonary function in lung disease [151] could reflect this aspect of ASMD. Indeed, the 6 minute walking distance is positively correlated with survival in IPF [152]. However, correlation between the 6MWT and pulmonary disease in ASMD is currently unknown as the test does not provide insight into the mechanisms of exercise limitation. In addition, the results can be affected by a variety of factors unrelated to cardiopulmonary status, including age, sex, height, and weight [151]. An advantage is that exercise tests allow a subjective parameter (i.e. stamina and dyspnea) to be translated to a score or number (for instance walked distance in a $6 \mathrm{MWT}$ ) that can be performed periodically and compared over time. The 6MWT is the procedure most studied in ASMD and related diseases such as IPF to objectively test exercise 
tolerance (additional + on tentative validation score, see Table 1).

\section{Conclusions and discussion}

This manuscript provides an overview of the histological, imaging and biochemical biomarkers of ASMD and assesses their usefulness in clinical practice. Perhaps not surprisingly, most parameters have not been properly validated for their capacity to substitute for relevant clinical endpoints. Based on the applied validation score, only diffusion capacity, spleen volume, platelet count and LDL-cholesterol score positively on all three criteria (i.e. (a) link to the pathophysiological process, (b) response to effective therapy and (c) correlation with clinical events). When feasibility is taken into account, the fibroscan to assess liver fibrosis, LSM and walked distance in six minutes are added to the most promising biomarkers of ASMD. A limitation of the scoring system is that "response to therapy" adds to the validation but has only been investigated in small human populations or mice. Several very sensitive biochemical markers such as LSM or LSM-509/PPCS, need to be further validated but may prove to be of high value as early response markers to therapy.

The markers that are discussed here are mostly markers that are supposed to reflect evident organ involvement, when structural changes have occurred already. However, markers that are of high interest would reflect changes early in disease progression and therefore be more predictive of irreversible disease. Of specific interest are for instance techniques that are able to demonstrate accumulation of storage material in liver, lungs and spleen, as well as techniques that can identify early stages of inflammation or fibrosis in these organs. Such markers would support the development of criteria for the initiation of therapy. In Gaucher disease for example, the window of opportunity for treatment is relatively wide, i.e. only in advanced stages, such as liver fibrosis, irreversible disease manifestations are present. In ASMD, this is currently unknown yet likely to be similar to Gaucher disease. Severe pulmonary involvement with fibrosis may occur even when liver and spleen are only moderately enlarged and vice versa [12]. Ideally, the risk for development of, for example, pulmonary fibrosis, could be predicted based upon disease markers as part of a risk based treatment algorithm. Here lies a major responsibility for physicians to team up with each other to establish causal relationships between disease markers and clinical endpoints in relation to treatment. Over the next years, olipudase alfa will probably become authorized and accessible for patients with ASMD: worldwide investment in a registry that not only collect effectiveness and safety data but has the ability for validation of disease markers will be the next challenge. The independent international Niemann-Pick registry (INPDR) can play an important role in this.

An important disease outcome has so far not been mentioned: parameters that reflect patient wellbeing and factors limiting daily activities. The focus in the current study has been on objective parameters that can be measured and expressed in numbers or scores. How disease manifestations, however, relate to performance status and quality of life is unknown. In ASMD, quality of life is mainly influenced by fatigue, dyspnea and pain. Psychosocial consequences of ASMD and effects on performance status have been reported [153,154]. Progression of disease for instance resulting in liver failure or pulmonary insufficiency obviously has an effect on the quality of life as well [155-158]. Although they do not meet the definition of a biomarker, questionnaires are a useful tool in assessing quality of life over time, because they are mutually comparable. To periodically assess healthrelated quality of life in ASMD patients, questionnaires such as the Short Form 36 Health Survey (also known as SF36) or Health Assessment Questionnaire (HAQ) can be used [159]. Again, such information should be captured in an independent registry. For the purpose of health technology assessment and national reimbursement policies, we expect that these outcomes will be of crucial importance as well.

In conclusion, this review provides an overview of potential biomarkers in ASMD and defines clinical endpoints to which these biomarkers should be validated. It is a starting point for research and validation of biomarkers for ASMD in the future. With the upcoming possibility of ERT, these biomarkers might aid in identifying patients who are at risk of developing major organ manifestations Moreover, these biomarkers might be useful in monitoring the rate of disease progression. This in turn might help to identify those patients who will benefit most from therapy in the future.

\section{Declarations}

Barbara Sjouke, Frédéric Vaz, Susan Goorden, André van Kuilenburg and Johannes Aerts have no competing interests to declare. Eline Eskes is as a sub-investigator involved in a pre-marketing study with Sanofi Genzyme. Carla Hollak is involved in premarketing studies with Sanofi, Protalix and Idorsia. This research did not receive any specific grant from funding agencies in the public, commercial, or notfor-profit sectors.

\section{References}

[1] E.H. Schuchman, R.J. Desnick, Types A and B Niemann-Pick disease, Mol, Genet. Metab. 120 (1-2) (2017) 27-33.

[2] C.R. Ferreira, W.A. Gahl, Lysosomal storage diseases, Transl. Sci. Rare Dis. 2 (1-2) (2017) 1-71.

[3] E.H. Schuchman, The pathogenesis and treatment of acid sphingomyelinase-deficient Niemann-Pick disease, J. Inherit. Metab. Dis. 30 (5) (2007) 654-663.

[4] R.O. Brady, J.N. Kanfer, M.B. Mock, D.S. Fredrickson, The metabolism of sphingomyelin. II. Evidence of an enzymatic deficiency in Niemann-Pick diseae, Proc. Natl. Acad. Sci. U. S. A. 55 (2) (1966) 366-369.

[5] E.H. Schuchman, Acid sphingomyelinase, cell membranes and human disease: lessons from Niemann-Pick disease, FEBS Lett. 584 (9) (2010) 1895-1900.

[6] K. Sandhoff, Metabolic and cellular bases of sphingolipidoses, Biochem. Soc. Trans. 41 (6) (2013) 1562-1568.

[7] P.J. Meikle, J.J. Hopwood, A.E. Clague, W.F. Carey, Prevalence of lysosomal storage disorders, JAMA. 281 (3) (1999) 249-254.

[8] S.D. Kingma, O.A. Bodamer, F.A. Wijburg, Epidemiology and diagnosis of lysosomal storage disorders; challenges of screening, Best Pract. Res. Clin. Endocrinol. Metab. 29 (2) (2015) 145-157.

[9] M. Acuna, P. Martinez, C. Moraga, X. He, M. Moraga, B. Hunter, et al., Epidemiological, clinical and biochemical characterization of the p.(Ala359Asp) SMPD1 variant causing Niemann-Pick disease type B, Eur. J. Hum. Genet. 24 (2) (2016) 208-213.

[10] C.M. Simonaro, J.H. Park, E. Eliyahu, N. Shtraizent, M.M. McGovern, E.H. Schuchman, Imprinting at the SMPD1 locus: implications for acid sphingomyelinase-deficient Niemann-Pick disease, Am. J. Hum. Genet. 78 (5) (2006) 865-870.

[11] M.M. McGovern, R. Avetisyan, B.J. Sanson, O. Lidove, Disease manifestations and burden of illness in patients with acid sphingomyelinase deficiency (ASMD), Orphanet. J. Rare Dis. 12 (1) (2017) 41.

[12] C.E. Hollak, E.S. de Sonnaville, D. Cassiman, G.E. Linthorst, J.E. Groener, E. Morava, et al., Acid sphingomyelinase (Asm) deficiency patients in the Netherlands and Belgium: disease spectrum and natural course in attenuated patients, Mol. Genet. Metab. 107 (3) (2012) 526-533.

[13] M.P. Wasserstein, R.J. Desnick, E.H. Schuchman, S. Hossain, S. Wallenstein, C. Lamm, et al., The natural history of type B Niemann-Pick disease: results from a 10-year longitudinal study, Pediatrics. 114 (6) (2004) e672-e677.

[14] O. Lidove, N. Belmatoug, R. Froissart, C. Lavigne, I. Durieu, K. Mazodier, et al., Acid sphingomyelinase deficiency (Niemann-Pick disease type B) in adulthood: a retrospective multicentric study of 28 adult cases, Rev. Med. Interne. 38 (5) (2017) 291-299.

[15] M.M. McGovern, C. Dionisi-Vici, R. Giugliani, P. Hwu, O. Lidove, Z. Lukacs, et al., Consensus recommendation for a diagnostic guideline for acid sphingomyelinase deficiency, Genet. Med. 19 (9) (2017) 967-974.

[16] S. Zampieri, M. Filocamo, A. Pianta, S. Lualdi, L. Gort, M.J. Coll, et al., SMPD1 mutation update: database and comprehensive analysis of published and novel variants, Hum. Mutat. 37 (2) (2016) 139-147.

[17] L. Pick, Gaucher's disease and similar diseases (type Niemann lipoid cell splenohepatomegaly and spleen diabetic lipoid cell hypoplasia); Der Morbus Gaucher und die ihm ähnlichen Krankheiten (die lipoidzellige Splenohepato-megalie Typus Niemann und die diabetische Lipoidzellen-hypoplasie der Milz), Ergebnisse der Inneren Medizin und Kinderheil-kunde. 29 (1926) 519-627.

[18] J. Stirnemann, N. Belmatoug, F. Camou, C. Serratrice, R. Froissart, C. Caillaud, et al., A review of gaucher disease pathophysiology, clinical presentation and treatments, Int. J. Mol. Sci. 18 (2) (2017).

[19] R.O. Brady, J.N. Kanfer, R.M. Bradley, D. Shapiro, Demonstration of a deficiency of glucocerebroside-cleaving enzyme in Gaucher's disease, J. Clin. Invest. 45 (7) (1966) 1112-1115.

[20] E. Klenk, Uber die natur der phosphatide der milz bei Niemann-Pickchen 
Krankheit, Z. Physiol. Chem. 229 (1934) 151-156.

[21] A. Rosenberg, E. Chargaff, A reinvestigation of the cerebroside deposited in Gaucher's disease, J. Biol. Chem. 233 (6) (1958) 1323-1326.

[22] US National Library of Medicine, Efficacy, Safety, Pharmacodynamic, and Pharmacokinetics Study of Olipudase Alfa in Patients With Acid Sphingomyelinase Deficiency (ASCEND), Available from, 2019. https://clinicaltrials.gov/ct2/show/ NCT02004691 term $=$ olipudase\&cond $=$ Niemann-Pick + Diseases\&rank $=1$.

[23] J.M. Murray, A.M. Thompson, A. Vitsky, M. Hawes, W.L. Chuang, J. Pacheco, et al., Nonclinical safety assessment of recombinant human acid sphingomyelinase (rhASM) for the treatment of acid sphingomyelinase deficiency:the utility of animal models of disease in the toxicological evaluation of potential therapeutics, Mol. Genet. Metab. 114 (2) (2015) 217-225.

[24] M.M. McGovern, M.P. Wasserstein, B. Kirmse, W.L. Duvall, T. Schiano, B.L. Thurberg, et al., Novel first-dose adverse drug reactions during a phase I trial of olipudase alfa (recombinant human acid sphingomyelinase) in adults with Niemann-Pick disease type B (acid sphingomyelinase deficiency), Genet. Med. 18 (1) (2016) 34-40.

[25] B.L. Thurberg, M.P. Wasserstein, T. Schiano, F. O’Brien, S. Richards, G.F. Cox, et al., Liver and skin histopathology in adults with acid sphingomyelinase deficiency (Niemann-Pick disease type B), Am. J. Surg. Pathol. 36 (8) (2012) 1234-1246.

[26] M.P. Wasserstein, S.A. Jones, H. Soran, G.A. Diaz, N. Lippa, B.L. Thurberg, et al., Successful within-patient dose escalation of olipudase alfa in acid sphingomyelinase deficiency, Mol. Genet. Metab. 116 (1-2) (2015) 88-97.

[27] B.L. Thurberg, M.P. Wasserstein, S.A. Jones, T.D. Schiano, G.F. Cox, A.C. Puga, Clearance of hepatic sphingomyelin by Olipudase alfa is associated with improvement in lipid profiles in acid sphingomyelinase deficiency, Am. J. Surg. Pathol. 40 (9) (2016) 1232-1242.

[28] M.P. Wasserstein, G.A. Diaz, R.H. Lachmann, M.H. Jouvin, I. Nandy, A.J. Ji, et al., Olipudase alfa for treatment of acid sphingomyelinase deficiency (ASMD): safety and efficacy in adults treated for 30 months, J. Inherit. Metab. Dis. 41 (2018) 829-838.

[29] C.E. Hollak, M. de Fost, L. van Dussen, S. Vom Dahl, J.M. Aerts, Enzyme therapy for the treatment of type 1 Gaucher disease: clinical outcomes and dose - response relationships, Expert. Opin. Pharmacother. 10 (16) (2009) 2641-2652.

[30] N.W. Barton, R.O. Brady, J.M. Dambrosia, A.M. Di Bisceglie, S.H. Doppelt, S.C. Hill, et al., Replacement therapy for inherited enzyme deficiency-macrophage-targeted glucocerebrosidase for Gaucher's disease, N. Engl. J. Med. 324 (21) (1991) 1464-1470.

[31] G. Biomarkers Definitions Working, Biomarkers and surrogate endpoints: preferred definitions and conceptual framework, Clin. Pharmacol. Ther. 69 (3) (2001) 89-95.

[32] BEST (Biomarkers, EndpointS, and other Tools), Resource, Silver Spring (MD) (2016).

[33] T.M. Cox, Biomarkers in lysosomal storage diseases, in: A. Mehta, M. Beck, G. Sunder-Plassmann (Eds.), Fabry Disease: Perspectives from 5 Years of FOS, Oxford, 2006.

[34] European Medicines Agency (EMA), Qualification of Novel Methodologies for Drug Development: Guidance to Applicants 2014, Available from https://www. ema.europa.eu/documents/regulatory-procedural-guideline/qualification-novelmethodologies-drug-development-guidance-applicants_en.pdf.

[35] Food and Drug Administration (FDA), CDER Biomarker Qualification Program, Available from, 2018. https://www.fda.gov/drugs/drug-development-toolqualification-programs/cder-biomarker-qualification-program.

[36] J.P.A. Ioannidis, P.M.M. Bossuyt, Waste, leaks, and failures in the biomarker pipeline, Clin. Chem. 63 (5) (2017) 963-972.

[37] P.J. Monaghan, S.J. Lord, A. St John, S. Sandberg, C.M. Cobbaert, L. Lennartz, et al., Biomarker development targeting unmet clinical needs, Clin. Chim. Acta 460 (2016) 211-219.

[38] L.A. Boven, M. van Meurs, R.G. Boot, A. Mehta, L. Boon, J.M. Aerts, et al., Gaucher cells demonstrate a distinct macrophage phenotype and resemble alternatively activated macrophages, Am. J. Clin. Pathol. 122 (3) (2004) 359-369.

[39] M.J. Ferraz, W.W. Kallemeijn, M. Mirzaian, D. Herrera Moro, A. Marques P. Wisse, et al., Gaucher disease and Fabry disease: new markers and insights in pathophysiology for two distinct glycosphingolipidoses, Biochim. Biophys. Acta 1841 (5) (2014) 811-825.

[40] D. Schuppan, N.H. Afdhal, Liver cirrhosis, Lancet. 371 (9615) (2008) 838-851.

[41] N. Chalasani, Z. Younossi, J.E. Lavine, A.M. Diehl, E.M. Brunt, K. Cusi, et al., The diagnosis and management of non-alcoholic fatty liver disease: practice guideline by the American Gastroenterological Association, American Association for the Study of Liver Diseases, and American College of Gastroenterology, Gastroenterology, 142 (7) (2012) 1592-1609.

[42] S. Yatsuji, E. Hashimoto, M. Tobari, M. Taniai, K. Tokushige, K. Shiratori, Clinical features and outcomes of cirrhosis due to non-alcoholic steatohepatitis compared with cirrhosis caused by chronic hepatitis C, J. Gastroenterol. Hepatol. 24 (2) (2009) 248-254.

[43] A.G. Nicholson, R. Florio, D.M. Hansell, R.M. Bois, A.U. Wells, P. Hughes, et al., Pulmonary involvement by Niemann-Pick disease. A report of six cases, Histopathology. 48 (5) (2006) 596-603.

[44] H.M.P. Freitas, A.D. Mancano, R.S. Rodrigues, B. Hochhegger, P. Torres, D. Escuissato, et al., Niemann-Pick disease type B: HRCT assessment of pulmonary involvement, J. Bras. Pneumol. 43 (6) (2017) 451-455.

[45] R.K. Putman, H. Hatabu, T. Araki, G. Gudmundsson, W. Gao, M. Nishino, et al., Association between interstitial lung abnormalities and all-cause mortality, JAMA. 315 (7) (2016) 672-681.

[46] E. Fernandez Fabrellas, R. Peris Sanchez, C. Sabater Abad, S.G. Juan, Prognosis and follow-up of idiopathic pulmonary fibrosis, Med. Sci. (Basel) 6 (2) (2018).

[47] Z. Chen, Z. Chen, S. Wu, X. Wang, Niemann-Pick disease resulting in spontaneous splenic rupture in an adult: report of a case, Surg. Today 38 (5) (2008) 473-475.

[48] P.J. Dawson, G. Dawson, Adult Niemann-Pick disease with sea-blue histiocytes in the spleen, Hum. Pathol. 13 (12) (1982) 1115-1120.

[49] O. Lidove, L. Le Fevre, N. Goasguen, M. Jamali, L. Vercellino, M. Garnier, et al., Acid sphingomyelinase deficiency and spleen trauma: splenectomy or not splenectomy? Rev. Med. Interne. 36 (9) (2015) 619-622.

[50] P. Rodon, J.P. Ramain, P. Bruandet, A. Piedon, J. Akli, J. Penot, Type B NiemannPick disease and sea-blue histiocytes syndrome, Rev. Med. Interne. 12 (4) (1991) 299-302.

[51] R.G. Simoes, H. Maia, Niemann-Pick type B in adulthood, BMJ Case Rep. 2015 (2015).

[52] E. Benedetti, A. Proietti, P. Miccoli, F. Basolo, E. Ciancia, P.A. Erba, et al., Contrast-enhanced ultrasonography in nodular splenomegaly associated with type B Niemann-Pick disease: an atypical hemangioma enhancement pattern, $\mathrm{J}$. Ultrasound. 12 (3) (2009) 85-92.

[53] L. van Dussen, M. Biegstraaten, M.G. Dijkgraaf, C.E. Hollak, Modelling Gaucher disease progression: long-term enzyme replacement therapy reduces the incidence of splenectomy and bone complications, Orphanet. J. Rare Dis. 9 (2014) 112.

[54] P.R. Fleshner, A.H. Aufses Jr., G.A. Grabowski, R. Elias, A 27-year experience with splenectomy for Gaucher's disease, Am. J. Surg. 161 (1) (1991) 69-75.

[55] M.M. McGovern, N. Lippa, E. Bagiella, E.H. Schuchman, R.J. Desnick, M.P. Wasserstein, Morbidity and mortality in type B Niemann-Pick disease, Genet. Med. 15 (8) (2013) 618-623.

[56] D. Cassiman, S. Packman, B. Bembi, H.B. Turkia, M. Al-Sayed, M. Schiff, et al., Cause of death in patients with chronic visceral and chronic neurovisceral acid sphingomyelinase deficiency (Niemann-Pick disease type B and B variant): literature review and report of new cases, Mol. Genet. Metab. 118 (3) (2016) 206-213.

[57] H. Ishii, T. Takahashi, M. Toyono, M. Tamura, K. Harada, M. Yoshida, et al., Acid sphingomyelinase deficiency: cardiac dysfunction and characteristic findings of the coronary arteries, J. Inherit. Metab. Dis. 29 (1) (2006) 232-234.

[58] A.M. Lever, J.B. Ryder, Cor pulmonale in an adult secondary to Niemann-Pick disease, Thorax. 38 (11) (1983) 873-874.

[59] M. Wasserstein, J. Godbold, M.M. McGovern, Skeletal manifestations in pediatric and adult patients with Niemann Pick disease type B, J. Inherit. Metab. Dis. 36 (1) (2013) 123-127.

[60] T. Takahashi, K. Akiyama, M. Tomihara, T. Tokudome, F. Nishinomiya, Y. Tazawa, et al., Heterogeneity of liver disorder in type B Niemann-Pick disease, Hum. Pathol. 28 (3) (1997) 385-388.

[61] M. Ziol, A. Handra-Luca, A. Kettaneh, C. Christidis, F. Mal, F. Kazemi, et al., Noninvasive assessment of liver fibrosis by measurement of stiffness in patients with chronic hepatitis C, Hepatology. 41 (1) (2005) 48-54.

[62] M. Webb, A. Zimran, T. Dinur, O. Shibolet, S. Levit, D.M. Steinberg, et al., Are transient and shear wave elastography useful tools in Gaucher disease? Blood Cells Mol. Dis. 68 (2018) 143-147.

[63] M.E. Rinella, Nonalcoholic fatty liver disease: a systematic review, JAMA. 313 (22) (2015) 2263-2273.

[64] M. Maximos, F. Bril, P. Portillo Sanchez, R. Lomonaco, B. Orsak, D. Biernacki, et al., The role of liver fat and insulin resistance as determinants of plasma aminotransferase elevation in nonalcoholic fatty liver disease, Hepatology. 61 (1) (2015) 153-160.

[65] O. Lidove, F. Sedel, F. Charlotte, R. Froissart, M.T. Vanier, Cirrhosis and liver failure: expanding phenotype of acid sphingomyelinase-deficient niemann-pick disease in adulthood, JIMD Rep. 15 (2015) 117-121.

[66] N. Guillemot, C. Troadec, T.B. de Villemeur, A. Clement, B. Fauroux, Lung disease in Niemann-Pick disease, Pediatr. Pulmonol. 42 (12) (2007) 1207-1214.

[67] B. Gulhan, U. Ozcelik, F. Gurakan, S. Gucer, D. Orhan, G. Cinel, et al., Different features of lung involvement in Niemann-Pick disease and Gaucher disease, Respir. Med. 106 (9) (2012) 1278-1285.

[68] G. Rossi, A. Cavazza, P. Spagnolo, S. Bellafiore, E. Kuhn, P. Carassai, et al., The role of macrophages in interstitial lung diseases: Number 3 in the Series "Pathology for the clinician" Edited by Peter Dorfmuller and Alberto Cavazza, Eur. Respir. Rev. 26 (145) (2017).

[69] A. Herve, S. Marchand-Adam, A. Fabre, M.P. Debray, D.P. Germain, B. Crestani, et al., Niemann-Pick disease type B identified following an episode of bronchopneumonia, Rev. Mal. Respir. 25 (7) (2008) 861-866.

[70] D.S. Mendelson, M.P. Wasserstein, R.J. Desnick, R. Glass, W. Simpson, G. Skloot, et al., Type B Niemann-Pick disease: findings at chest radiography, thin-section CT, and pulmonary function testing, Radiology. 238 (1) (2006) 339-345.

[71] P. Faverio, A. Stainer, F. De Giacomi, S. Gasperini, S. Motta, F. Canonico, et al., Molecular pathways and respiratory involvement in lysosomal storage diseases, Int. J. Mol. Sci. 20 (2) (2019).

[72] J. Ahuja, J.P. Kanne, C.A. Meyer, S.N. Pipavath, R.A. Schmidt, J.O. Swanson, et al. Histiocytic disorders of the chest: imaging findings, Radiographics. 35 (2) (2015) 357-370.

[73] M.J. Chung, K.S. Lee, T. Franquet, N.L. Muller, J. Han, O.J. Kwon, Metabolic lung disease: imaging and histopathologic findings, Eur. J. Radiol. 54 (2) (2005) 233-245.

[74] F.M. von Ranke, H.M. Pereira Freitas, A.D. Mancano, R.S. Rodrigues, B. Hochhegger, D. Escuissato, et al., Pulmonary involvement in Niemann-Pick disease: a state-of-the-art review, Lung. 194 (4) (2016) 511-518.

[75] M.M. McGovern, M.P. Wasserstein, R. Giugliani, B. Bembi, M.T. Vanier, E. Mengel, et al., A prospective, cross-sectional survey study of the natural history of Niemann-Pick disease type B, Pediatrics. 122 (2) (2008) e341-e349. 
[76] P. Lipinski, L. Kuchar, E.Y. Zakharova, G.V. Baydakova, A. Lugowska, A. TylkiSzymanska, Chronic visceral acid sphingomyelinase deficiency (Niemann-Pick disease type B) in 16 polish patients: long-term follow-up, Orphanet. J. Rare Dis. 14 (1) (2019) 55.

[77] P.J. Vinholt, The role of platelets in bleeding in patients with thrombocytopenia and hematological disease, Clin. Chem. Lab. Med. 57 (2019) 1808-1817.

[78] M.M. McGovern, T. Pohl-Worgall, R.J. Deckelbaum, W. Simpson, D. Mendelson, R.J. Desnick, et al., Lipid abnormalities in children with types A and B Niemann Pick disease, J. Pediatr. 145 (1) (2004) 77-81.

[79] A.C. Jansen, E.S. van Aalst-Cohen, M.W. Tanck, M.D. Trip, P.J. Lansberg, A.H. Liem, et al., The contribution of classical risk factors to cardiovascular disease in familial hypercholesterolaemia: data in 2400 patients, J. Intern. Med. 256 (6) (2004) 482-490.

[80] J.C. van Capelleveen, A.E. Bochem, S.M. Boekholdt, S. Mora, R.C. Hoogeveen, C.M. Ballantyne, et al., Association of High-Density Lipoprotein-Cholesterol Versus Apolipoprotein A-I With Risk of Coronary Heart Disease: The European Prospective Investigation Into Cancer-Norfolk Prospective Population Study, the Atherosclerosis Risk in Communities Study, and the Women's Health Study, J. Am. Heart Assoc. 6 (8) (2017).

[81] M. de Fost, M. Langeveld, R. Franssen, B.A. Hutten, J.E. Groener, E. de Groot, et al., Low HDL cholesterol levels in type I Gaucher disease do not lead to an increased risk of cardiovascular disease, Atherosclerosis. 204 (1) (2009) 267-272.

[82] C. Hollak, M. Maas, E. Akkerman, A. den Heeten, H. Aerts, Dixon quantitative chemical shift imaging is a sensitive tool for the evaluation of bone marrow responses to individualized doses of enzyme supplementation therapy in type 1 Gaucher disease, Blood Cells Mol. Dis. 27 (6) (2001) 1005-1012.

[83] L.A. Johnson, B.E. Hoppel, E.L. Gerard, S.P. Miller, S.H. Doppelt, G.C. Zirzow, et al., Quantitative chemical shift imaging of vertebral bone marrow in patients with Gaucher disease, Radiology. 182 (2) (1992) 451-455.

[84] L. van Dussen, E.M. Akkerman, C.E. Hollak, A.J. Nederveen, M. Maas, Evaluation of an imaging biomarker, Dixon quantitative chemical shift imaging, in Gaucher disease: lessons learned, J. Inherit. Metab. Dis. 37 (6) (2014) 1003-1011.

[85] W.L. Chuang, J. Pacheco, S. Cooper, M.M. McGovern, G.F. Cox, J. Keutzer, et al., Lyso-sphingomyelin is elevated in dried blood spots of Niemann-Pick B patients, Mol. Genet. Metab. 111 (2) (2014) 209-211.

[86] L. Kuchar, J. Sikora, M.E. Gulinello, H. Poupetova, A. Lugowska, V. Malinova, et al., Quantitation of plasmatic lysosphingomyelin and lysosphingomyelin-509 for differential screening of Niemann-Pick a/B and C diseases, Anal. Biochem. 525 (2017) 73-77.

[87] G. Polo, A.P. Burlina, T.B. Kolamunnage, M. Zampieri, C. Dionisi-Vici, P. Strisciuglio, et al., Diagnosis of sphingolipidoses: a new simultaneous measurement of lysosphingolipids by LC-MS/MS, Clin. Chem. Lab. Med. 55 (3) (2017) 403-414.

[88] F. Deodato, S. Boenzi, R. Taurisano, M. Semeraro, G. Catesini, R. Carrozzo, et al., The impact of biomarkers analysis in the diagnosis of Niemann-Pick C disease and acid sphingomyelinase deficiency, Clin. Chim. Acta. 486 (2018) 387-394.

[89] M. Pettazzoni, R. Froissart, C. Pagan, M.T. Vanier, S. Ruet, P. Latour, et al., LC-MS/ MS multiplex analysis of lysosphingolipids in plasma and amniotic fluid: a novel tool for the screening of sphingolipidoses and Niemann-Pick type C disease, PLoS One 12 (7) (2017) e0181700.

[90] M. Voorink-Moret, S.M.I. Goorden, A.B.P. van Kuilenburg, F.A. Wijburg, G. der Vlugt JMM, F.S. Beers-Stet, et al., Rapid screening for lipid storage disorders using biochemical markers. Expert center data and review of the literature, Mol. Genet. Metab. 123 (2) (2018) 76-84.

[91] G. Polo, A.P. Burlina, E. Ranieri, F. Colucci, L. Rubert, A. Pascarella, et al., Plasma and dried blood spot lysosphingolipids for the diagnosis of different sphingolipidoses: a comparative study, Clin. Chem. Lab. Med. 57 (2019) 1863-1874.

[92] A.K. Giese, H. Mascher, U. Grittner, S. Eichler, G. Kramp, J. Lukas, et al., A novel, highly sensitive and specific biomarker for Niemann-Pick type C1 disease, Orphanet. J. Rare Dis. 10 (2015) 78.

[93] R. Sidhu, Y. Mondjinou, M. Qian, H. Song, A.B. Kumar, X. Hong, et al., N-acyl-Ophosphocholineserines: structures of a novel class of lipids that are biomarkers for Niemann-Pick C1 disease, J. Lipid Res. 60 (2019) 1410-1424.

[94] J.M. Aerts, J.E. Groener, S. Kuiper, W.E. Donker-Koopman, A. Strijland, R. Ottenhoff, et al., Elevated globotriaosylsphingosine is a hallmark of Fabry disease, Proc. Natl. Acad. Sci. U. S. A. 105 (8) (2008) 2812-2817.

[95] N. Dekker, L. van Dussen, C.E. Hollak, H. Overkleeft, S. Scheij, K. Ghauharali, et al., Elevated plasma glucosylsphingosine in Gaucher disease: relation to phenotype, storage cell markers, and therapeutic response, Blood. 118 (16) (2011) e118-e127.

[96] M.J. Ferraz, A.R. Marques, M.D. Appelman, M. Verhoek, A. Strijland, M. Mirzaian, et al., Lysosomal glycosphingolipid catabolism by acid ceramidase: formation of glycosphingoid bases during deficiency of glycosidases, FEBS Lett. 590 (6) (2016) $716-725$.

[97] N. Kaissarian, J. Kang, L. Shu, M.J. Ferraz, J.M. Aerts, J.A. Shayman, Dissociation of globotriaosylceramide and impaired endothelial function in alpha-galactosidase-A deficient EA.hy926 cells, Mol. Genet. Metab. 125 (4) (2018) 338-344.

[98] M.D. Sanchez-Nino, D. Carpio, A.B. Sanz, M. Ruiz-Ortega, S. Mezzano, A. Ortiz, Lyso-Gb3 activates Notch1 in human podocytes, Hum. Mol. Genet. 24 (20) (2015) 5720-5732.

[99] M.D. Sanchez-Nino, A.B. Sanz, S. Carrasco, M.A. Saleem, P.W. Mathieson, J.M. Valdivielso, et al., Globotriaosylsphingosine actions on human glomerular podocytes: implications for Fabry nephropathy, Nephrol. Dial. Transplant. 26 (6) (2011) 1797-1802.

[100] F. Weidemann, M.D. Sanchez-Nino, J. Politei, J.P. Oliveira, C. Wanner, D.G. Warnock, et al., Fibrosis: a key feature of Fabry disease with potential therapeutic implications, Orphanet. J. Rare Dis. 8 (2013) 116.

[101] L. Choi, J. Vernon, O. Kopach, M.S. Minett, K. Mills, P.T. Clayton, et al., The Fabry disease-associated lipid Lyso-Gb3 enhances voltage-gated calcium currents in sensory neurons and causes pain, Neurosci. Lett. 594 (2015) 163-168.

[102] P.K. Mistry, J. Liu, L. Sun, W.L. Chuang, T. Yuen, R. Yang, et al., Glucocerebrosidase 2 gene deletion rescues type 1 Gaucher disease, Proc. Natl. Acad. Sci. U. S. A. 111 (13) (2014) 4934-4939.

[103] M.K. Pandey, T.A. Burrow, R. Rani, L.J. Martin, D. Witte, K.D. Setchell, et al., Complement drives glucosylceramide accumulation and tissue inflammation in Gaucher disease, Nature. 543 (7643) (2017) 108-112.

[104] M.K. Pandey, G.A. Grabowski, J. Kohl, An unexpected player in Gaucher disease: the multiple roles of complement in disease development, Semin. Immunol. 37 (2018) 30-42.

[105] Y.V. Taguchi, J. Liu, J. Ruan, J. Pacheco, X. Zhang, J. Abbasi, et al., Glucosylsphingosine promotes alpha-Synuclein pathology in mutant GBA-associated Parkinson's disease, J. Neurosci. 37 (40) (2017) 9617-9631.

[106] H.M.T. Vissers, I. Campuzano, J. Langridge, H. Aerts, Serum profiling; the analysis of therapeutic proteins and diagnostic markers for lysosomal storage disorders by LC-MS, Mol. Cell. Proteomics 4 (8) (2005).

[107] A. Gomez-Munoz, N. Presa, A. Gomez-Larrauri, I.G. Rivera, M. Trueba, M. Ordonez, Control of inflammatory responses by ceramide, sphingosine 1phosphate and ceramide 1-phosphate, Prog. Lipid Res. 61 (2016) 51-62.

[108] B. Aguilera, K. Ghauharali-van der Vlugt, M.T. Helmond, J.M. Out, W.E. DonkerKoopman, J.E. Groener, et al., Transglycosidase activity of chitotriosidase: improved enzymatic assay for the human macrophage chitinase, J. Biol. Chem. 278 (42) (2003) 40911-40916.

[109] C.E. Hollak, S. van Weely, M.H. van Oers, J.M. Aerts, Marked elevation of plasma chitotriosidase activity. A novel hallmark of Gaucher disease, J. Clin. Invest. 93 (3) (1994) 1288-1292.

[110] R.G. Boot, M.J. van Breemen, W. Wegdam, R.R. Sprenger, S. de Jong, D. Speijer, et al., Gaucher disease: a model disorder for biomarker discovery, Expert Rev. Proteomics. 6 (4) (2009) 411-419.

[111] J.J. Sheth, F.J. Sheth, N.J. Oza, P.S. Gambhir, U.P. Dave, R.C. Shah, Plasma chitotriosidase activity in children with lysosomal storage disorders, Indian J. Pediatr. 77 (2) (2010) 203-205.

[112] M. Ries, E. Schaefer, T. Luhrs, L. Mani, J. Kuhn, M.T. Vanier, et al., Critical assessment of chitotriosidase analysis in the rational laboratory diagnosis of children with Gaucher disease and Niemann-Pick disease type A/B and C, J. Inherit. Metab. Dis. 29 (5) (2006) 647-652.

[113] J. Brinkman, F.A. Wijburg, C.E. Hollak, J.E. Groener, M. Verhoek, S. Scheij, et al., Plasma chitotriosidase and CCL18: early biochemical surrogate markers in type B Niemann-Pick disease, J. Inherit. Metab. Dis. 28 (1) (2005) 13-20.

[114] S. Kadali, A. Kolusu, S. Sunkara, M.R. Gummadi, J. Undamatla, Clinical evaluation of chitotriosidase enzyme activity in Gaucher and Niemann Pick A/B diseases: a retrospective study from India, Clin. Chim. Acta 457 (2016) 8-11.

[115] A. Wajner, K. Michelin, M.G. Burin, R.F. Pires, M.L. Pereira, R. Giugliani, et al., Biochemical characterization of chitotriosidase enzyme: comparison between normal individuals and patients with Gaucher and with Niemann-Pick diseases, Clin. Biochem. 37 (10) (2004) 893-897.

[116] N.V. Olkhovych, Chitotriosidase activity as additional biomarker in the diagnosis of lysosomal storage diseases, Ukr. Biochem. J. 88 (1) (2016) 69-78.

[117] R.G. Boot, G.H. Renkema, M. Verhoek, A. Strijland, J. Bliek, T.M. de Meulemeester, et al., The human chitotriosidase gene. Nature of inherited enzyme deficiency, J. Biol. Chem. 273 (40) (1998) 25680-25685.

[118] Y. Zhang, N. Kaminski, Biomarkers in idiopathic pulmonary fibrosis, Curr. Opin. Pulm. Med. 18 (5) (2012) 441-446.

[119] R.G. Boot, M. Verhoek, M. de Fost, C.E. Hollak, M. Maas, B. Bleijlevens, et al., Marked elevation of the chemokine CCL18/PARC in Gaucher disease: a novel surrogate marker for assessing therapeutic intervention, Blood. 103 (1) (2004) 33-39.

[120] K.L. Chang, W.L. Hwu, H.Y. Yeh, N.C. Lee, Y.H. Chien, CCL18 as an alternative marker in Gaucher and Niemann-Pick disease with chitotriosidase deficiency, Blood Cells Mol. Dis. 44 (1) (2010) 38-40.

[121] M.J.C. van der Lienden, P. Gaspar, R. Boot, J. Aerts, M. van Eijk, Glycoprotein nonmetastatic protein B: an emerging biomarker for lysosomal dysfunction in macrophages, Int. J. Mol. Sci. 20 (1) (2018).

[122] G. Kramer, W. Wegdam, W. Donker-Koopman, R. Ottenhoff, P. Gaspar, M. Verhoek, et al., Elevation of glycoprotein nonmetastatic melanoma protein B in type 1 Gaucher disease patients and mouse models, FEBS Open Bio. 6 (9) (2016) 902-913.

[123] V. Murugesan, J. Liu, R. Yang, H. Lin, A. Lischuk, G. Pastores, et al., Validating glycoprotein non-metastatic melanoma B (gpNMB, osteoactivin), a new biomarker of Gaucher disease, Blood Cells Mol. Dis. 68 (2018) 47-53.

[124] A.R. Marques, T.L. Gabriel, J. Aten, C.P. van Roomen, R. Ottenhoff, N. Claessen, et al., Gpnmb is a potential marker for the visceral pathology in Niemann-Pick type C disease, PLoS One 11 (1) (2016) e0147208.

[125] K. Hieshima, T. Imai, M. Baba, K. Shoudai, K. Ishizuka, T. Nakagawa, et al., A novel human CC chemokine PARC that is most homologous to macrophage-inflammatory protein-1 alpha/LD78 alpha and chemotactic for T lymphocytes, but not for monocytes, J. Immunol. 159 (3) (1997) 1140-1149.

[126] M.J. van Breemen, M. de Fost, M. Maas, M.G. Wiersma, C.E. Hollak, L.W. Poll, et al., Different dose-dependent correction of MIP-1beta and chitotriosidase during initial enzyme replacement therapy, J. Inherit. Metab. Dis. 32 (2) (2009) 274-279.

[127] E. Vargiami, M. Dimitriadou, M. Economou, A. Christoforidis, D.I. Zafeiriou, Longterm response in biochemical markers of bone turnover during enzyme replacement therapy in a case-series of patients with Gaucher disease type I from northern 
Greece, Hippokratia. 20 (2) (2016) 153-159.

[128] M.J. van Breemen, M. de Fost, J.S. Voerman, J.D. Laman, R.G. Boot, M. Maas, et al., Increased plasma macrophage inflammatory protein (MIP)-1alpha and MIP1beta levels in type 1 Gaucher disease, Biochim. Biophys. Acta 1772 (7) (2007) $788-796$.

[129] R. Dhami, M.A. Passini, E.H. Schuchman, Identification of novel biomarkers for Niemann-Pick disease using gene expression analysis of acid sphingomyelinase knockout mice, Mol. Ther. 13 (3) (2006) 556-564.

[130] N. Lin, H. Zhang, W. Qiu, J. Ye, L. Han, Y. Wang, et al., Determination of 7ketocholesterol in plasma by LC-MS for rapid diagnosis of acid SMase-deficient Niemann-Pick disease, J. Lipid Res. 55 (2) (2014) 338-343.

[131] F. Kannenberg, J.R. Nofer, E. Schulte, J. Reunert, T. Marquardt, M. Fobker, Determination of serum cholestane-3beta,5alpha,6beta-triol by gas chromatography-mass spectrometry for identification of Niemann-Pick type C (NPC) disease, J. Steroid Biochem. Mol. Biol. 169 (2017) 54-60.

[132] S. Pajares, A. Arias, J. Garcia-Villoria, J. Macias-Vidal, E. Ros, de las Heras J, et al. Cholestane-3beta,5alpha,6beta-triol: high levels in Niemann-Pick type C, cerebrotendinous xanthomatosis, and lysosomal acid lipase deficiency, J. Lipid Res. 56 (10) (2015) 1926-1935.

[133] F.D. Porter, D.E. Scherrer, M.H. Lanier, S.J. Langmade, V. Molugu, S.E. Gale, et al., Cholesterol oxidation products are sensitive and specific blood-based biomarkers for Niemann-Pick C1 disease, Sci Transl Med. 2 (56) (2010) $56 \mathrm{ra} 81$.

[134] M.T. Vanier, P. Gissen, P. Bauer, M.J. Coll, A. Burlina, C.J. Hendriksz, et al., Diagnostic tests for Niemann-Pick disease type C (NP-C): a critical review, Mol. Genet. Metab. 118 (4) (2016) 244-254.

[135] J. Reunert, M. Fobker, F. Kannenberg, I. Du Chesne, M. Plate, J. Wellhausen, et al., Rapid diagnosis of 83 patients with Niemann Pick type C disease and related cholesterol transport disorders by Cholestantriol screening, EBioMedicine. 4 (2016) 170-175.

[136] S. Boenzi, F. Deodato, R. Taurisano, B.M. Goffredo, C. Rizzo, C. Dionisi-Vici, Evaluation of plasma cholestane-3beta,5alpha,6beta-triol and 7-ketocholesterol in inherited disorders related to cholesterol metabolism, J. Lipid Res. 57 (3) (2016) 361-367.

[137] G. Klinke, M. Rohrbach, R. Giugliani, P. Burda, M.R. Baumgartner, C. Tran, et al., LC-MS/MS based assay and reference intervals in children and adolescents for oxysterols elevated in Niemann-Pick diseases, Clin. Biochem. 48 (9) (2015) 596-602.

[138] S. Boenzi, F. Deodato, R. Taurisano, D. Martinelli, D. Verrigni, R. Carrozzo, et al., A new simple and rapid LC-ESI-MS/MS method for quantification of plasma oxysterols as dimethylaminobutyrate esters. Its successful use for the diagnosis of Niemann-Pick type C disease, Clin. Chim. Acta 437 (2014) 93-100.

[139] M. Romanello, S. Zampieri, N. Bortolotti, L. Deroma, A. Sechi, A. Fiumara, et al., Comprehensive evaluation of plasma 7-Ketocholesterol and Cholestan-3beta,5alpha,6beta-Triol in an Italian cohort of patients affected by Niemann-Pick disease due to NPC1 and SMPD1 mutations, Clin. Chim. Acta 455 (2016) 39-45.

[140] Z. Pataj, G. Liebisch, G. Schmitz, S. Matysik, Quantification of oxysterols in human plasma and red blood cells by liquid chromatography high-resolution tandem mass spectrometry, J. Chromatogr. A 1439 (2016) 82-88.

[141] C.D. van Karnebeek, T. Mohammadi, N. Tsao, G. Sinclair, S. Sirrs, S. Stockler, et al., Health economic evaluation of plasma oxysterol screening in the diagnosis of Niemann-Pick type C disease among intellectually disabled using discrete event simulation, Mol. Genet. Metab. 114 (2) (2015) 226-232.

[142] T.G. Hammerschmidt, G. de Oliveira Schmitt Ribas, M.L. Saraiva-Pereira, M.P. Bonatto, R.G. Kessler, S. FTS, et al., Molecular and biochemical biomarkers for diagnosis and therapy monitorization of Niemann-Pick type C patients, Int. J. Dev. Neurosci. 66 (2018) 18-23.

[143] X. Jiang, R. Sidhu, F.D. Porter, N.M. Yanjanin, A.O. Speak, D.T. te Vruchte, et al., A sensitive and specific LC-MS/MS method for rapid diagnosis of Niemann-Pick C1 disease from human plasma, J. Lipid Res. 52 (7) (2011) 1435-1445.

[144] X. Jiang, R. Sidhu, L. Mydock-McGrane, F.F. Hsu, D.F. Covey, D.E. Scherrer, et al., Development of a bile acid-based newborn screen for Niemann-Pick disease type C, Sci. Transl. Med. 8 (337) (2016) 337ra63.

[145] L. Amigo, H. Mendoza, J. Castro, V. Quinones, J.F. Miquel, S. Zanlungo, Relevance of Niemann-Pick type C1 protein expression in controlling plasma cholesterol and biliary lipid secretion in mice, Hepatology. 36 (4 Pt 1) (2002) 819-828.

[146] F. Mazzacuva, P. Mills, K. Mills, S. Camuzeaux, P. Gissen, E.R. Nicoli, et al., Identification of novel bile acids as biomarkers for the early diagnosis of NiemannPick C disease, FEBS Lett. 590 (11) (2016) 1651-1662.

[147] T. Geberhiwot, A. Moro, A. Dardis, U. Ramaswami, S. Sirrs, M.P. Marfa, et al., Consensus clinical management guidelines for Niemann-Pick disease type C, Orphanet. J. Rare Dis. 13 (1) (2018) 50.

[148] M. Maekawa, M. Shimada, K. Ohno, M. Togawa, H. Nittono, T. Iida, et al., Focused metabolomics using liquid chromatography/electrospray ionization tandem mass spectrometry for analysis of urinary conjugated cholesterol metabolites from patients with Niemann-Pick disease type $\mathrm{C}$ and 3beta-hydroxysteroid dehydrogenase deficiency, Ann. Clin. Biochem. 52 (Pt 5) (2015) 576-587.

[149] M. Maekawa, A. Narita, I. Jinnoh, T. Iida, T. Marquardt, E. Mengel, et al., Diagnostic performance evaluation of sulfate-conjugated cholesterol metabolites as urinary biomarkers of Niemann-Pick disease type C, Clin. Chim. Acta 494 (2019) 58-63.

[150] M. Maekawa, Y. Misawa, A. Sotoura, H. Yamaguchi, M. Togawa, K. Ohno, et al., LC/ESI-MS/MS analysis of urinary 3beta-sulfooxy-7beta-N-acetylglucosaminyl-5cholen-24-oic acid and its amides: new biomarkers for the detection of NiemannPick type C disease, Steroids. 78 (10) (2013) 967-972.

[151] G.A. Heresi, R.A. Dweik, Strengths and limitations of the six-minute-walk test: a model biomarker study in idiopathic pulmonary fibrosis, Am. J. Respir. Crit. Care Med. 183 (9) (2011) 1122-1124.

[152] K.R. Flaherty, A.C. Andrei, S. Murray, C. Fraley, T.V. Colby, W.D. Travis, et al., Idiopathic pulmonary fibrosis: prognostic value of changes in physiology and sixminute-walk test, Am. J. Respir. Crit. Care Med. 174 (7) (2006) 803-809.

[153] G.F. Cox, L.A. Clarke, R. Giugliani, M.M. McGovern, Burden of illness in acid sphingomyelinase deficiency: a retrospective chart review of 100 patients, JIMD Rep. 41 (2018) 119-129.

[154] S.L. Henderson, W. Packman, S. Packman, Psychosocial aspects of patients with Niemann-Pick disease, type B, Am. J. Med. Genet. A 149A (11) (2009) 2430-2436.

[155] Z.M. Younossi, N. Boparai, L.L. Price, M.L. Kiwi, M. McCormick, G. Guyatt, Healthrelated quality of life in chronic liver disease: the impact of type and severity of disease, Am. J. Gastroenterol. 96 (7) (2001) 2199-2205.

[156] G. Marchesini, G. Bianchi, P. Amodio, F. Salerno, M. Merli, C. Panella, et al., Factors associated with poor health-related quality of life of patients with cirrhosis, Gastroenterology. 120 (1) (2001) 170-178.

[157] J.J. Swigris, W.G. Kuschner, S.S. Jacobs, S.R. Wilson, M.K. Gould, Health-related quality of life in patients with idiopathic pulmonary fibrosis: a systematic review, Thorax. 60 (7) (2005) 588-594.

[158] J. De Vries, M. Drent, Quality of life and health status in interstitial lung diseases, Curr. Opin. Pulm. Med. 12 (5) (2006) 354-358.

[159] B. Bruce, J.F. Fries, The Stanford health assessment questionnaire: dimensions and practical applications, Health Qual. Life Outcomes 1 (2003) 20. 